\title{
Photoinduced hole-transfer in semiconducting polymer/low-bandgap cyanine dye blends: evidence for unit charge separation quantum yield $\dagger$
}

\author{
Fernando A. Castro, ${ }^{* a}$ Hadjar Benmansour, $\ddagger^{a}$ Jacques-E. Moser, ${ }^{b}$ \\ Carlos F. O. Graeff, ${ }^{c}$ Frank Nüesch ${ }^{a}$ and Roland Hany ${ }^{a}$
}

Received 13th May 2009, Accepted 16th July 2009

First published as an Advance Article on the web 14th August 2009

DOI: $10.1039 / \mathrm{b} 909512 \mathrm{~h}$

Power-conversion efficiencies of organic heterojunction solar cells can be increased by using semiconducting donor-acceptor materials with complementary absorption spectra extending to the near-infrared region. Here, we used continuous wave fluorescence and absorption, as well as nanosecond transient absorption spectroscopy to study the initial charge transfer step for blends of a donor poly( $p$-phenylenevinylene) derivative and low-band gap cyanine dyes serving as electron acceptors. Electron transfer is the dominant relaxation process after photoexcitation of the donor. Hole transfer after cyanine photoexcitation occurs with an efficiency close to unity up to dye concentrations of $\sim 30 \mathrm{wt} \%$. Cyanines present an efficient self-quenching mechanism of their fluorescence, and for higher dye loadings in the blend, or pure cyanine films, this process effectively reduces the hole transfer. Comparison between dye emission in an inert polystyrene matrix and the donor matrix allowed us to separate the influence of self-quenching and charge transfer mechanisms. Favorable photovoltaic bilayer performance, including high open-circuit voltages of $\sim 1 \mathrm{~V}$ confirmed the results from optical experiments. The characteristics of solar cells using different dyes also highlighted the need for balanced adjustment of the energy levels and their offsets at the heterojunction when using low-bandgap materials, and accentuated important effects of interface interactions and solid-state packing on charge generation and transport.

\section{Introduction}

The potential of cheap photovoltaics is fueling the interest in organic semiconductors. ${ }^{1-4}$ State-of-the-art devices are made from a combination of electron-donor and electron-acceptor (D-A) materials, sandwiched between metallic electrodes. ${ }^{5}$ Photoexcitation of either of the two components leads to an exciton that can dissociate into free charge carriers at the D-A interface. ${ }^{6}$ After charge separation, electrons and holes are transported via drift and diffusion processes to the electrodes, where they are collected, giving rise to an electric current. ${ }^{2}$

Device efficiency is determined by the short-circuit current $\left(J_{\mathrm{sc}}\right)$, the open-circuit voltage $\left(V_{\mathrm{oc}}\right)$, and the fill factor $(\mathrm{FF})$ via $\eta=\left(J_{\mathrm{sc}} V_{\mathrm{oc}} \mathrm{FF}\right) / P$, where $P$ is the incident optical power. The $\mathrm{FF}$ is a measure of the ability to transport and extract charges

\footnotetext{
${ }^{a}$ Empa, Swiss Federal Laboratories for Materials Testing and Research, Laboratory for Functional Polymers, Überlandstr. 129. CH-8600 Dübendorf, Switzerland.

E-mail: fernando.araujo@empa.ch; Fax:+4144 823-4012.

Tel: $+4144823-4786$

${ }^{b}$ Photochemical Dynamics Group, Institute of Chemical Sciences and Engineering, Ecole Polytechnique Fédérale de Lausanne, CH-1015 Lausanne, Switzerland

${ }^{c}$ Departamento de Física-FC-UNESP, Av. Luiz Edmundo Carrijo Coube, 14-01, 17033-360 Bauru, Brazil

$\dagger$ Electronic supplementary information (ESI) available: Simulation of fluorescence spectra; comparison of PL/absorbance spectral overlap and PL quenching dependences on dye loading; and comparison of electronic-vibronic coupling in different polymer matrices. See DOI: $10.1039 / \mathrm{b} 909512 \mathrm{~h}$

$\ddagger$ Present address: Total S.A. - Gaz \& Energies Nouvelles, 2 Place Jean Millier - La Défense 6, 92400 Coubevoie, France.
}

when the applied voltage approaches $V_{\text {oc }} ; J_{\text {sc }}$ is determined by the fraction of absorbed photons from the incident sunlight as well as by the ability to create charges via exciton dissociation at the D-A interface. Consensus has now been reached that $V_{\text {oc }}$ correlates with the energy difference $\left(E_{\Delta}\right)$ between the highest occupied molecular orbital (HOMO) of the donor and the lowest unoccupied molecular orbital (LUMO) of the acceptor. $^{7-9}$

It follows that both $J_{\mathrm{sc}}$ and $V_{\mathrm{oc}}$ depend on the donor and acceptor energy levels and their offsets at the heterojunction (for illustration, see Fig. 1c). A high $V_{\text {oc }}$ requires a high $E_{\Delta}$. However, this implies that the HOMO-HOMO $\left(E_{\mathrm{HH}}\right)$ and LUMO-LUMO $\left(E_{\mathrm{LL}}\right)$ energy gaps decrease, which results in a lower driving force for electron transfer, inefficient exciton dissociation, and a lowered $J_{\mathrm{sc}}$. Therefore, a compromise must be found to optimize the product $J_{\mathrm{sc}} V_{\mathrm{oc}}{ }^{10}$

Tuning the energy levels to ensure optimal values for $E_{\Delta}$, $E_{\mathrm{HH}}$ and $E_{\mathrm{LL}}$ at the same time gets increasingly difficult when so-called low-bandgap materials are involved. Most polymers and small molecules used so far have bandgaps $>2 \mathrm{eV}$, thus they are not able to absorb light beyond $600 \mathrm{~nm}$. This means that around $60 \%$ of the solar photons are not absorbed and do not contribute to the first necessary step in the current generation process. In particular, fullerene $\mathrm{C}_{60}$ and its derivatives, which are widely used electron-acceptors, have low absorption outside the UV region. ${ }^{5}$ Therefore, intensive efforts have been made to synthesize semiconducting materials that absorb at longer wavelengths and collect a larger fraction of the incident sunlight. ${ }^{11-14}$ 
a)

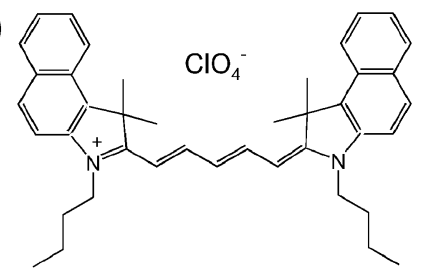

CyA

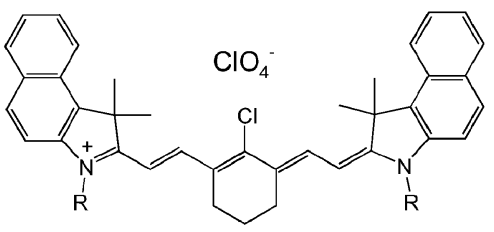

CyBs: $\mathrm{R}=-\mathrm{CH}_{2} \mathrm{CH}_{3}$

CyBI: $\quad \mathrm{R}=-\left(\mathrm{CH}_{2}\right)_{17} \mathrm{CH}_{3}$

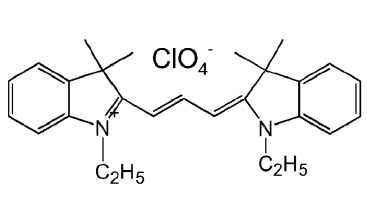

Cyc

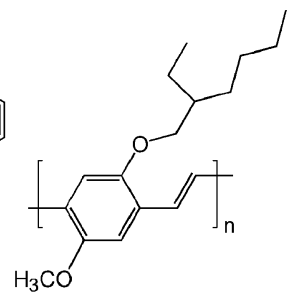

MEH-PPV b)

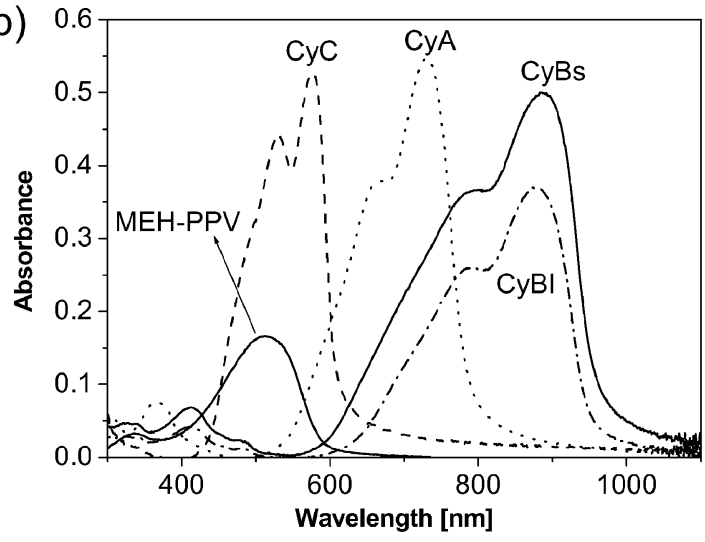

c)

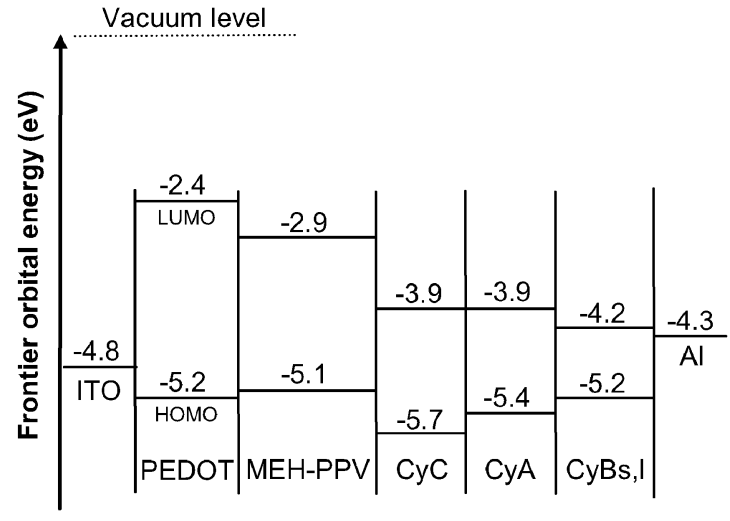

Fig. 1 (a) Molecular structures and (b) absorbance spectra of the electron-donor MEH-PPV, and the electron-acceptor cyanine dyes CyA, CyBs (with short alkyl side chains), CyBl (with long alkyl side chains) and CyC. For all dyes, perchlorate was the counter anion. (c) Energy levels of the frontier molecular orbitals relative to the vacuum level.

For this purpose, cyanine dyes are also of interest. ${ }^{15}$ Cyanines are charged polymethine dyes that benefit from high extinction coefficients and a tunable absorption spectrum by varying the number of double bonds. Different from most small molecules, cyanines are easily processed from solution which makes them attractive for technological applications. Recently, low-bandgap cyanine dyes have been investigated for photoconductive ${ }^{16}$ and photovoltaic applications up to 800 nm. $^{15}$ Their photophysical properties have been extensively investigated in solution ${ }^{17-19}$ or when anchored to silver halide ${ }^{20}$ or $\mathrm{TiO}_{2}$ surfaces, ${ }^{21}$ however, solid organic films and blends have received little attention. ${ }^{22,23}$

In this work, we investigate the charge-transfer characteristics of four cyanine dyes in solid-state blends with poly[2-methoxy-5-(2'-ethylhexyloxy)-1,4-phenylenevinylene] (MEH-PPV) and with polystyrene (PS) using absorption and fluorescence spectroscopy, and correlate these characteristics with the D-A energy levels at the heterojunction. Transient absorption measurements confirmed our results and indicated unit hole-charge separation quantum yield. We fabricated double layer photovoltaic devices using the cyanines as electron acceptors and MEH-PPV as donor. We report on high open-circuit voltages $(\sim 0.9-1.0 \mathrm{~V})$ for these devices, and discuss the influence of different redox levels and lengths of alkyl side chains on hole transfer and solar cell parameters.

\section{Results}

Fig. 1 shows the chemical structures of the molecules used, their absorbance spectra, and the relevant orbital energies relative to the vacuum level. According to the energy diagram, one expects efficient electron transfer after excitation of the polymer (LUMO of MEH-PPV $=-2.9 \mathrm{eV}$ ) to all dyes (LUMO of dyes $=-3.9$ or $-4.2 \mathrm{eV}$ ), since $E_{\mathrm{LL}} \geq 1 \mathrm{eV}$. On the other hand, the energy difference, $E_{\mathrm{HH}}$, between the dye HOMO levels and the polymer decreases substantially in the order $\mathrm{CyC}$, CyA, CyBs,1, that is, with decreasing cyanine bandgap. Consequently, the driving force for hole transfer after cyanine excitation decreases in this direction. ${ }^{10}$

Fig. 2 shows the absorbance spectra of thin films of MEH-PPV : CyA (2a) and MEH-PPV : CyBl (2b) blends for different dye concentrations. In both cases, the absorbance spectra of the blends are a composition of the individual components (MEH-PPV from $\sim 400-600 \mathrm{~nm}$, CyA from $\sim 600-780 \mathrm{~nm}$, and $\mathrm{CyBl}$ from $\sim 650-950 \mathrm{~nm}$, see Fig. 1b). No new absorption band appeared which indicates that no chargetransfer complexes are formed in the ground state. The spectra display a bathochromic shift $(\sim 20 \mathrm{~nm})$ as well as an increase of the full-width at half-maximum (FWHM) of the dye bands with increasing concentrations. Indeed, the dye absorbance spectra approach the lambda-max $(731 \mathrm{~nm}$ for CyA, $878 \mathrm{~nm}$ for $\mathrm{CyBl}$ ) and FWHM values (138 nm for CyA, $191 \mathrm{~nm}$ for $\mathrm{CyBl}$ ) of the pure films for dye contents of $2.25 \times 10^{-3} \mathrm{~mol} \mathrm{~g}^{-1}$ polymer (compare with Fig. 1b). On the other hand, for larger polymer concentrations, and thus with decreasing percentage of dye in the blend, the MEH-PPV absorption band shifts slightly $(\sim 20 \mathrm{~nm})$ to smaller wavelengths.

Fig. 3a shows fluorescence spectra (PL) of thin films of MEH-PPV : CyA. The emission spectrum of a pure MEH-PPV film is shown for comparison. The excitation wavelength was at $480 \mathrm{~nm}$, where only the polymer is directly excited. The polymer emission is efficiently quenched with increasing dye 

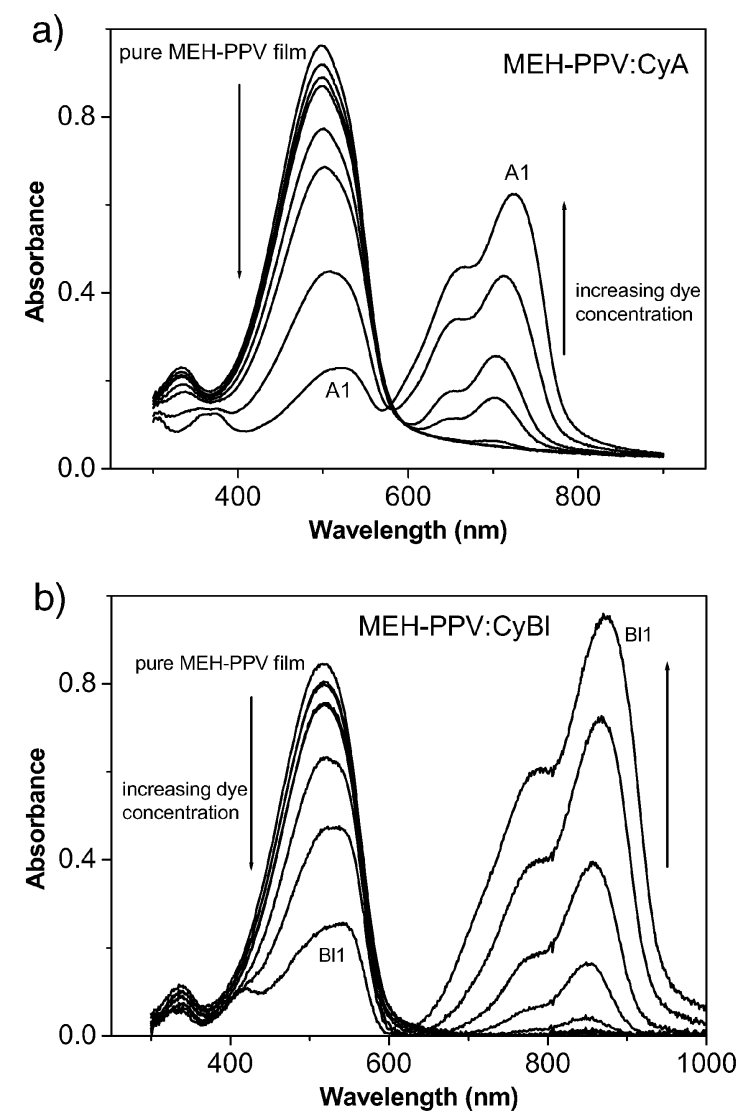

Fig. 2 Absorbance spectra of (a) MEH-PPV:CyA and (b) MEH-PPV: $\mathrm{CyBl}$ blend films with varying dye concentration: $2.25 \times 10^{-3}(\mathrm{~A} 1, \mathrm{~B} 11), 6.40 \times 10^{-4}, 1.65 \times 10^{-4}, 4.65 \times 10^{-5}$, $1.50 \times 10^{-5}, 4.50 \times 10^{-6}$ and $1.50 \times 10^{-6} \mathrm{~mol} \mathrm{~g}^{-1}$ of polymer. Arrows indicate the direction of increasing dye concentration.

content. At the same time, a new PL band developed above $700 \mathrm{~nm}$ due to CyA emission. This suggests that energy transfer from excited MEH-PPV to CyA is taking place; a consequence of the large overlap between polymer emission and dye absorption. Although this effect competes with electron transfer, it does not exclude its occurrence (see below).

MEH-PPV:CyBl films were also measured. Fig. 3b compares the quenching efficiency for both dyes. Since the overlap between polymer emission and $\mathrm{CyBl}$ absorption is small, energy transfer from MEH-PPV is unlikely in this case, and no cyanine PL was observed (not shown). This therefore indicates that, for $\mathrm{CyBl}$, the most probable quenching mechanism is electron transfer. The LUMO level of CyA is $0.3 \mathrm{eV}$ higher in energy than that of $\mathrm{CyB}$, but both are at least $1 \mathrm{eV}$ below the LUMO of MEH-PPV. Thus, we argue that electron transfer from the polymer to CyA also occurs.

To examine the hole transfer mechanism, similar PL experiments were carried out after selective dye excitation. Since the dyes absorb at longer wavelengths than MEH-PPV, energy transfer to MEH-PPV would be endothermic and, therefore, unlikely. Fig. 4a shows the emission spectra of MEH-PPV : CyA blends excited at $650 \mathrm{~nm}$, and that of a pure CyA thin film for comparison. The dye film is only weakly emissive, and the PL increases as the CyA is diluted into the polymer matrix. These results suggest a PL self-quenching
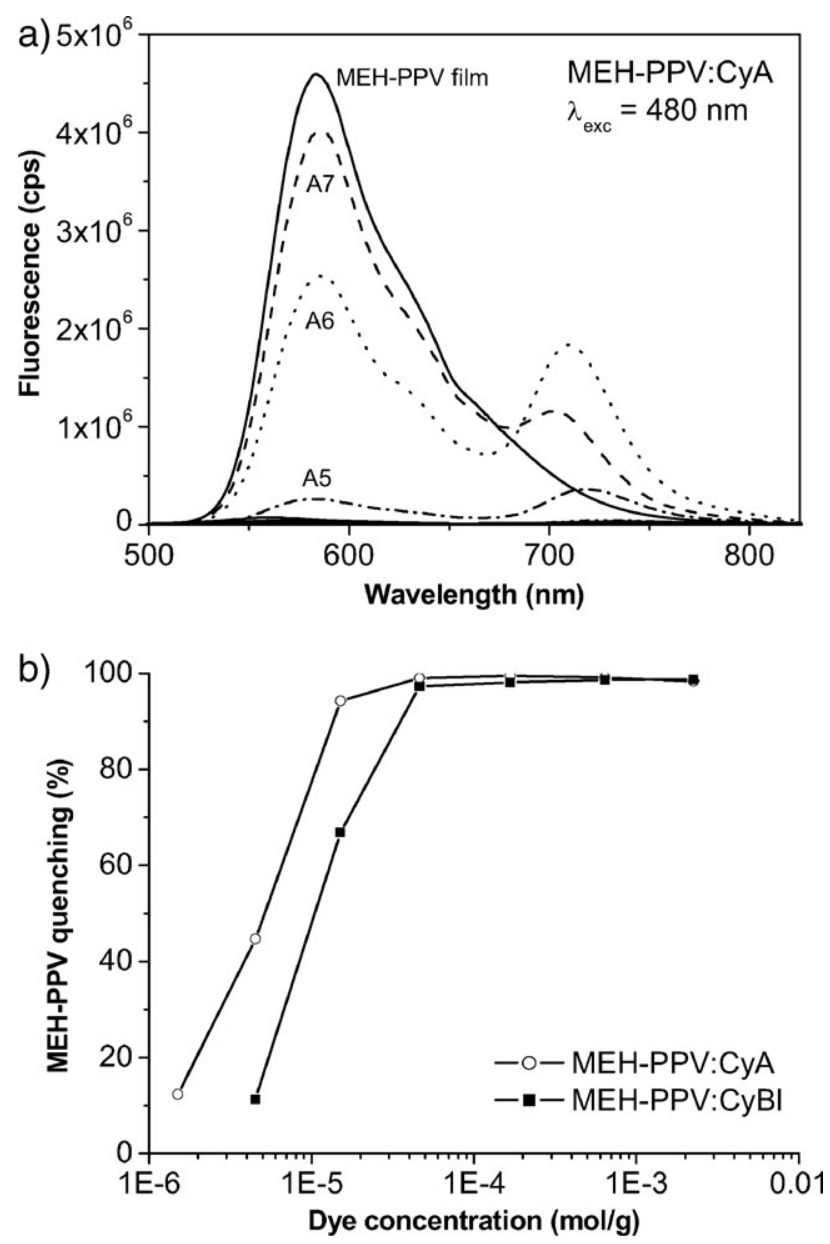

Fig. 3 Fluorescence spectra of (a) MEH-PPV:CyA blend films with varying concentration of dye: $2.25 \times 10^{-3}, 6.40 \times 10^{-4}$, $1.65 \times 10^{-4}, 4.65 \times 10^{-5}, 1.50 \times 10^{-5}(\mathrm{~A} 5), 4.50 \times 10^{-6}$ (A6) and $1.50 \times 10^{-6}(\mathrm{~A} 7) \mathrm{mol} \mathrm{g}^{-1}$ of polymer. Films were excited at $480 \mathrm{~nm}$, where only the polymer absorbs. In (b) the percentage of MEH-PPV emission quenching is plotted against dye concentration for both MEH-PPV : CyA and MEH-PPV : CyBl blend films.

process as the dye molecules pack closer with increasing concentration. We prepared blends of CyA and polystyrene (PS) in the same relative concentrations as used for MEH-PPV. PS is an inert polymer and allows investigation of the effect of diluting CyA in a polymer matrix separately, without the interference of quenching by hole transfer to MEH-PPV.

Fig. $4 \mathrm{~b}$ shows the results of these PL measurements in PS: CyA blends, again with the excitation wavelength at $650 \mathrm{~nm}$. The PL intensity continuously increased with decreasing dye concentration, corroborating the self-quenching effect. The position of the emission peak also changed with dye concentration. A red shift of the zero-phonon peak occurred from $0.1 \mathrm{wt} \%$ up to $1 \mathrm{wt} \%$, and, for high concentrations, the emission seems to split into two components. At the same time, the change in position of the vibrational peak $(\sim 827 \mathrm{~nm})$ is small. The same trend was observed in the dye: MEH-PPV system. These changes in peak position can be explained in part by cyanine self-absorption, which is enhanced by a small Stokes-shift $\left(\sim 420 \mathrm{~cm}^{-1}\right)$ and the strong CyA extinction coefficient $\left(\varepsilon(680 \mathrm{~nm})=190000 \mathrm{~L} \mathrm{~mol}^{-1} \mathrm{~cm}^{-1}\right.$ in methanol). 
a)

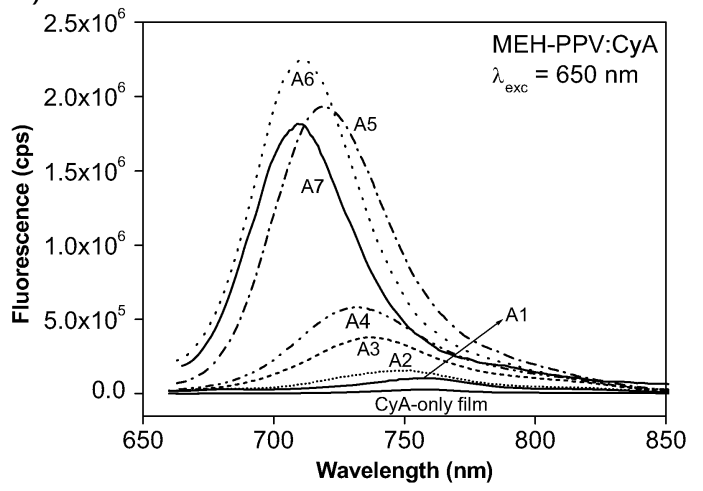

b)

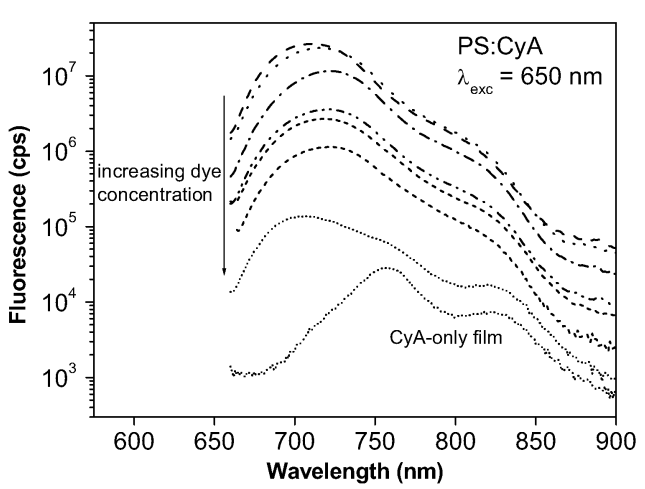

c)

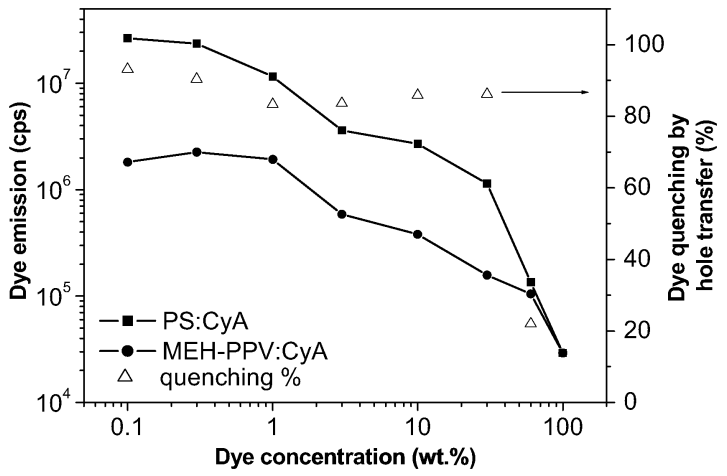

Fig. 4 Fluorescence spectra of (a) MEH-PPV : CyA and (b) PS : CyA blend films with varying concentration of dye: $0.1 \mathrm{wt} \%$ (A7), $0.3 \mathrm{wt} \%$ (A6), $1 \mathrm{wt} \%$ (A5), $3 \mathrm{wt} \%$ (A4), $10 \mathrm{wt} \%$ (A3), $30 \mathrm{wt} \%$ (A2) and $60 \mathrm{wt} \%$ (A1). Films were excited at $650 \mathrm{~nm}$, where only the dye absorbs. In (c) we compare the intensity of dye emission in both polymer matrices (left) and estimate the dye emission quenching due to hole transfer (right, triangles).

For the further analysis, we assume the dye - matrix interactions to be equal for MEH-PPV and PS. As the common basic mechanisms that quench the PL of cyanines, we consider radiative and non-radiative decay for both polymers, including self-quenching and re-absorption. In addition, for MEH-PPV, exciton dissociation via charge transfer takes place. By comparing the dye PL intensities in both polymers (Fig. 4c), we can then estimate the relative importance of hole transfer to MEH-PPV. Since no energy transfer from the excited dye to the polymer is expected, we conclude that hole transfer from excited CyA to MEH-PPV occurs with an efficiency of approximately $85 \%$ in blends with dye loadings between 1 and $30 \mathrm{wt} \%$. Only for the highest dye loading of $60 \mathrm{wt} \%$ does the charge transfer efficiency drop, from over $80 \%$ to approximately $20 \%$. This indicates that the excited state deactivation rate effectively competes with hole transfer.

The possible hole transfer from $\mathrm{CyBl}$ and $\mathrm{CyC}$ to MEH-PPV was also studied. In the case of $\mathrm{CyBl}$, no fluorescence could be observed in the solid state, even at very low concentrations in a PS blend, or when $\mathrm{CyBl}$ was dissolved in chlorobenzene for concentrations higher than $\sim 2 \times 10^{-2} \mathrm{~g} \mathrm{~L}^{-1}$. For $\mathrm{CyC}$, both MEH-PPV and PS blend films were investigated (data not shown). Due to the strong overlap of the MEH-PPV and $\mathrm{CyC}$ absorption spectra, it was not possible to excite each material individually and to selectively observe the change in fluorescence. We observed a strong quenching of the PL for increasing dye concentration in MEH-PPV, and a self-quenching behavior in PS blends similar to CyA.

To support the results of high photoinduced hole transfer quantum yield obtained by steady state fluorescence measurements, we measured the nanosecond transient absorptions of a pure CyA film and a blend sample of MEH-PPV:CyA $(30 \mathrm{wt} \%)$. Fig. 5 shows the temporal profiles of transient absorbance changes of these samples recorded at $526 \mathrm{~nm}$ upon nanosecond pulsed laser excitation at $650 \mathrm{~nm}$. The inset shows the signal obtained for the dye-polymer blend at a shorter time-scale and a single exponential fit curve with a rate constant of $4.5 \times 10^{6} \mathrm{~s}^{-1}$. Upon pulsed laser excitation of pure CyA films at $\lambda=650 \mathrm{~nm}$, bleaching signals were observed over a wavelength domain extending from $580-850 \mathrm{~nm}$ and roughly corresponding to the absorption spectrum of the dye. In addition, a positive absorbance change was observed at shorter wavelengths, with a maximum at $\lambda=526 \mathrm{~nm}$ (Fig. 5, upper curve). The appearance of this transient absorbance signal took place within the 5 ns laser pulse duration and, hence, could not be time-resolved with the setup that was used. The temporal evolution of the absorbance recorded at $526 \mathrm{~nm}$ was characterized by a multi-exponential decay, with typical rate constants of the order of $2.3 \times 10^{6} \mathrm{~s}^{-1}$

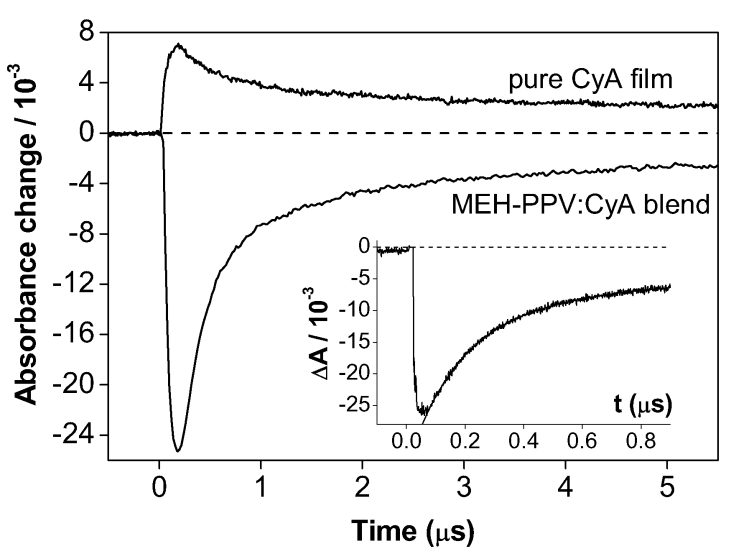

Fig. 5 Temporal profiles of transient absorbance changes recorded at $\lambda=526 \mathrm{~nm}$ of pure CyA and MEH-PPV:CyA blend films upon nanosecond pulsed laser excitation at $\lambda=650 \mathrm{~nm}$. The inset shows the signal obtained for the dye-polymer blend at a shorter time-scale. The smooth curve superimposed on experimental data results from a single exponential fit with $k=4.5 \times 10^{6} \mathrm{~s}^{-1}$. 


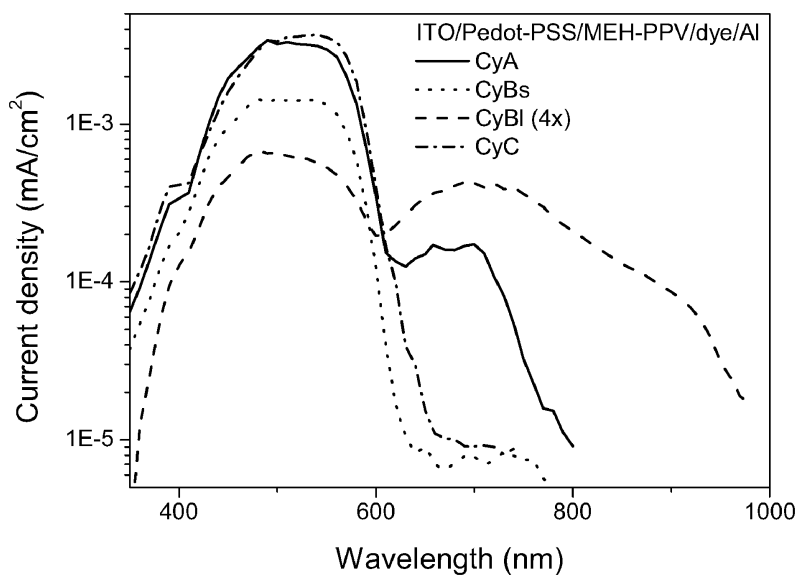

Fig. 6 Photocurrent-density from ITO/PEDOT-PSS/MEH-PPV/ dye/Al double layer devices. For better visualization, the current value of the $\mathrm{CyBl}$ device was multiplied by 4 .

$(55 \%), 3.8 \times 10^{5} \mathrm{~s}^{-1}(30 \%)$ and $5.7 \times 10^{2} \mathrm{~s}^{-1}(15 \%)$. The decay of the signal recorded at $526 \mathrm{~nm}$ was mirrored by the recovery of the ground state absorption at $700 \mathrm{~nm}$. Different from the pure CyA dye film, a very large bleaching signal was observed at $526 \mathrm{~nm}$ for MEH-PPV : CyA blends (Fig. 5, lower curve) measured under the same conditions, which will be discussed in detail in the next section.

Finally, we fabricated double layer dye : MEH-PPV photovoltaic devices and investigated their photocurrent spectra (Fig. 6). The spectra clearly show that the main part of the current is always produced in the wavelength region where MEH-PPV absorbs, but with different efficiencies. For CyA, $\mathrm{CyC}$ and $\mathrm{CyBl}$, photocurrent due to hole transfer is also observed. No such contribution occurs for $\mathrm{CyBs}$, although the only structural difference between $\mathrm{CyBl}$ and $\mathrm{CyBs}$ is the length of the alkyl side chain (see Fig. 1a).

The power conversion efficiencies (not shown) of these devices were low $(\eta<0.1 \%)$ when cells were excited with half-sun simulated AM1.5G light $\left(47 \mathrm{~mW} \mathrm{~cm}^{-2}\right)$. Note that all measurements were made for as prepared cells, before biasing the device. Application of bias can displace the mobile ions present in the cyanine molecules across the heterojunction and drastically affect the device performance. Details of such ionic effects were recently described. ${ }^{24}$

\section{Discussion}

The interactions between the blend components have profound effects on thin film optical properties. Due to intermolecular polymer interaction and aggregation, it is expected that the absorption spectrum of MEH-PPV shifts to longer wavelength with increasing concentration. ${ }^{25,26}$ However, for MEH-PPV: dye films the contrary is observed (Fig. 2). We argue that a decrease of the polymer fraction with a concomitant increase of the dye concentration leads to a strong increase in polarity. This induces a bathochromic shift of the polymer $\pi-\pi^{*}$ transition larger than the aggregation-induced wavelength change. The strong tendency of cyanines to aggregate ${ }^{27}$ explains the increase in absorption wavelength with increasing dye concentration.
Our transient absorption measurements in pure CyA films showed multi-exponential signal decay. The observed slow kinetics, where the shortest time constant exceeds $400 \mathrm{~ns}$, excludes the possibility that the observed transient signal could arise from the absorption of the singlet excited states of the dye, whose lifetimes in the solid film lie in the picosecond time frame. Rather, self-quenching processes of CyA, such as triplet-triplet annihilation, yielding both $\mathrm{CyA}^{+}$and $\mathrm{CyA}^{-}$ radical species may be at the origin of the recorded absorbance signal. Oxidized states of cyanine dyes are typically characterized by absorption spectra that are blue-shifted with regard to the ground state absorption. ${ }^{28}$ The transient absorbance recorded at $526 \mathrm{~nm}$ is therefore very likely to be due to the $\mathrm{CyA}^{+}$radical dication, which decayed by recombining with $\mathrm{CyA}^{-}$species. Self-quenching behavior was also observed in cw PL measurements of cyanine: PS blends, where the signal was quenched by up to three orders of magnitude with increasing dye concentration. To test the importance of selfabsorption in the fluorescence quenching phenomenon, we simulated PL spectra (ESI $\dagger$ ) according to a literature model. ${ }^{17}$ The simulated and experimental spectra agreed only for blends with very low dye concentrations (below $1 \mathrm{wt} \%$ ), meaning that self-absorption alone is not able to explain the pronounced quenching. A decrease of PL intensity with increasing chromophore concentration has been observed in other dyes as well. ${ }^{17,18,29}$ Most photophysical studies were made in solution ${ }^{19,30}$ and often these conclusions cannot be transferred to the condensed state. In solution, one of the main non-radiative pathways for excited-state deactivation is cis-trans photo-isomerization and/or the formation of a twisted intramolecular charge transfer state. ${ }^{29,30}$ No charge transfer state signature could be observed in our absorbance spectra. Also, photo-isomerization is inhibited due to the rigidity in the film. ${ }^{31}$ Therefore, we rule out these processes as the cause of PL self-quenching in the cyanines. We speculate that cyanine dimer/aggregate formation possibly accounts for the self-quenching. Larger, H-type aggregates also act as non-radiative quenching centers, ${ }^{32}$ however, no signatures of such species were observed in the absorbance spectra.

Fig. 3 shows that the MEH-PPV emission is quenched as soon as $0.1 \mathrm{wt} \%$ of CyA is added to the film blend, with a yield of $\sim 99 \%$ for dye concentrations higher than $1 \mathrm{wt} \%$. The appearance of a new emission band $(>700 \mathrm{~nm})$ indicates that energy is transferred from MEH-PPV to the dye. This could be either due to radiative or long-range non-radiative energy transfer. To estimate the importance of radiative transfer, we evaluated the spectral overlap defined by the normalized donor emission and the absorption spectrum of the acceptor (see Experimental). ${ }^{33}$ If radiative energy transfer was the main reason for PL quenching, the dependence of PL intensity on dye concentration should follow the trend of the spectral overlap defined above. This expectation contradicts the experiment (Fig. 3b and ESI $\dagger$ ), since a strong increase in PL quenching occurs for concentrations between 0.1 and $1 \mathrm{wt} \%$, a range where the transmission at the maximum dye absorption amounts to $\sim 90 \%$. However, high fluorescence quenching is observed, ruling out radiative energy transfer as the main mechanism. For a MEH-PPV density of $\sim 1 \mathrm{~g} \mathrm{~cm}^{-3}$, 
we estimate that for CyA concentrations above $1 \mathrm{wt} \%$, the primary photoexcitations are able to reach an acceptor molecule within the MEH-PPV exciton diffusion length $\left(\sim 5 \mathrm{~nm}^{34}\right)$. Thus, by comparing the MEH-PPV PL quenching efficiency in Fig. $3 \mathrm{~b}$ for $\mathrm{CyA}$ and $\mathrm{CyBl}$, we argue that the energy transfer observed at these relatively low cyanine concentrations is due to Förster transfer. As the mean distance between cyanine molecules decreases, electron transfer may become more important and even dominate the quenching mechanism. This would imply that the electron transfer is faster than the energy transfer process, a justified estimate since the photoinduced electron transfer from MEH-PPV to fullerenes is known to be in the sub-picosecond time scale. $^{35}$

By comparing cyanine PL quenching as a function of concentration in two different polymer matrices (Fig. 4c), we estimated a high quantum yield of photoinduced hole transfer ( $\sim 85 \%$ ) from the CyA dye to MEH-PPV. This was confirmed by transient absorption spectroscopy. Films of CyA only and MEH-PPV : CyA blends (30 wt\%) were submitted to laser flash photolysis under identical experimental conditions. In both cases, a transient bleaching of the dye ground state was observed at $700 \mathrm{~nm}$. However, in contrast to the dye-only film case where the kinetics were multi-exponential, the recovery of the initial absorbance in the MEH-PPV : CyA blend appeared to take place with biphasic kinetics. Fitting the experimental data with a double exponential rate law yielded a rate constant of $4.5 \times 10^{6} \mathrm{~s}^{-1}$ for the initial phase, accounting for $\mathrm{ca} .90 \%$ of the signal amplitude. The remaining $\sim 10 \%$ of the signal was recovered in the millisecond time scale, with a rate constant of the order of $1.5 \times 10^{3} \mathrm{~s}^{-1}$.

The oxidized MEH-PPV radical cation spectrum was reported to cover a very broad wavelength domain extending from $1200 \mathrm{~nm}$ in the NIR down to the blue region in the visible. ${ }^{36}$ This absorption overlaps in part with that of the ground state of CyA below $850 \mathrm{~nm}$. In addition, the triplet state of MEH-PPV is also expected to absorb light in the near infrared around $850 \mathrm{~nm} .{ }^{37}$ These various spectral properties then render the direct interpretation of transient signals at wavelengths larger than $750 \mathrm{~nm}$ rather difficult. Hence, it was decided that the photoredox reaction involving MEH-PPV would be followed by measuring transient absorbance signals at $\lambda \sim 520 \mathrm{~nm}$. While films consisting of pure CyA dye displayed a positive transient absorbance peaking at $\lambda=526 \mathrm{~nm}$, a very large bleaching signal was indeed observed under the same conditions for MEH-PPV : CyA blends, with a maximum amplitude at $\lambda=515 \mathrm{~nm}$ (Fig. 5, lower curves). This particular probe wavelength corresponds to the absorption maximum of the MEH-PPV polymer (Fig. 2). The CyA dye spectrum being precisely characterized by a minimum around $500 \mathrm{~nm}$, the observed negative signal is clearly due to the bleaching of the polymer ground state absorption. The bleaching signal recorded at $526 \mathrm{~nm}$ was found to follow kinetics identical to those obtained at $700 \mathrm{~nm}$, and a biphasic recovery of the initial absorbance with $k=4.5 \times 10^{6} \mathrm{~s}^{-1}(80-90 \%)$ and $1.5 \times 10^{3} \mathrm{~s}^{-1}$ (10-20\%) was indeed observed again.

The CyA absorption is clearly red-shifted compared to that of MEH-PPV, with minimal overlap between both spectra.
Thus, energy transfer from the cyanine excited state to MEH-PPV is not thermodynamically feasible. Moreover, the temporal behavior of the bleaching signal at $526 \mathrm{~nm}$ is not compatible with the expected short lifetime of the polymer's excited state. $^{38}$ Therefore, based on this observation and on the quenching of the singlet state emission, it is inferred that the bleaching of MEH-PPV absorption at $526 \mathrm{~nm}$ can only be originating from the charge transfer reaction

$$
{ }^{1} \mathrm{CyA}^{*}+\mathrm{MEH}-\mathrm{PPV} \rightarrow \mathrm{CyA}^{-}+{\mathrm{MEH}-\mathrm{PPV}^{+} .}^{+}
$$

The quantum yield of the hole transfer process (eqn (1)) can be approximately evaluated from the values of the extinction coefficient, $\varepsilon$, of the polymer's repeating unit at the probe wavelength, $\lambda=526 \mathrm{~nm}$, the absorbance, $A_{\text {dye }}$, of CyA in the film at the excitation wavelength, $\lambda_{\mathrm{exc}}=650 \mathrm{~nm}$, and the incident laser energy fluence, $F$. Several samples were measured, typically with $A_{\text {dye }}=0.26$ and $F=1.33 \times 10^{-3} \mathrm{~J} \mathrm{~cm}^{-2}$, yielding an experimental transient absorbance change $\Delta A$ $(526 \mathrm{~nm})=2.6 \times 10^{-2}$ at the end of the excitation pulse. Taking into account a value $\varepsilon(526 \mathrm{~nm})=1.56 \times 10^{7} \mathrm{~cm}^{2} \mathrm{~mol}^{-1}$ per repeating unit of MEH-PPV, calculated for a thin polymer film with a density of $1 \mathrm{~g} \mathrm{~cm}^{-3}$, and assuming the Beer-Lambert law is indeed applicable within the film, the quantum yield $\Phi_{\mathrm{CT}}$ of the charge separation process can be calculated as:

$$
\Phi_{\mathrm{CT}}=\frac{\Delta A N_{\mathrm{A}} h c}{\varepsilon F\left[1-\exp \left(-A_{\mathrm{dye}}\right)\right] \lambda_{\mathrm{exc}}},
$$

where $N_{\mathrm{A}}$ is Avogadro's number, $h$ Planck's constant, and $c$ the speed of light.

We obtain a unit quantum yield $\left(\Phi_{\mathrm{CT}}=1.00 \pm 0.07\right)$ for charge separation evidenced by transient absorbance spectrometry measurements, where the uncertainty is mainly given by the determination of the number of exciting photons and the MEH-PPV extinction coefficient. This result is in excellent agreement with the $\sim 85 \%$ PL quenching efficiency deduced from cw fluorescence analysis of MEH-PPV : CyA films loaded with up to $30 \mathrm{wt} \%$ cyanine. Although pulsed laser experiments were conducted with only CyA blends, it is expected that this conclusion can be extended to the other dye molecules, whose emission was also efficiently quenched when mixed with the polymer.

Based on the estimated high charge transfer quantum yields, we prepared bilayer photovoltaic devices and measured their photocurrent spectra, as shown in Fig. 6. We note here that no bulk heterojunctions were fabricated since high-concentration cyanine:MEH-PPV blends are not fully miscible and phase separation occurs during spin coating. ${ }^{39}$ This would add intricate morphology issues to our analysis. For all dyes, the current is generated in the wavelength region where MEH-PPV absorbs, meaning that the electron transfer is favorable, as expected due to the energy differences $E_{\mathrm{LL}} \geq 1 \mathrm{eV}$. For $\mathrm{CyC}$, the hole transfer after cyanine absorption in the 400-600 nm region (see Fig. 1b) also contributes to the current, and the maximum is slightly increased and shifted to longer wavelengths, as compared to CyA.

The contribution of CyA to current generation (600-800 nm) is much smaller than expected from optical spectroscopy 
experiments alone. This is a consequence of the fact that the measured photocurrent does not only depend on the initial charge transfer efficiency; the created charges must be extracted as well. Charge mobility is key for the transport and collection efficiency at the electrodes. Also, the small D-A interface area of the bilayer leads to photoexcited cyanines far away from the heterojunction. For small diffusion lengths, these excitons do not contribute to charge generation and decay by other processes. Both the cyanines' charge mobilities and exciton diffusion lengths are not known at this moment, and are the subject of further studies. Finally, the bilayer device configuration actually refers to the situation of high cyanine concentration (see Fig. 4c) with efficient PL quenching and reduced hole transfer.

The low-bandgap dyes, CyBs and CyBl, differ only in the length of the alkyl side chains (Fig. 1a), but the corresponding spectra show distinct differences. The photocurrent after electron transfer from MEH-PPV using $\mathrm{CyBl}$ is more than eight times smaller than with CyBs; however, photocurrent in the dye absorption region is not produced at all using CyBs (Fig. 6). The long alkyl side chains in $\mathrm{CyBl}$ increase the distance between cyanine cores, leading to reduced electron transport through the acceptor layer. Also, if the octadecyl group acts as a spacer between donor and acceptor at the heterojunction, the initial charge transfer process might be adversely affected, thereby reducing the electronic D-A coupling. ${ }^{40}$ This probably explains the lower photocurrent in the MEH-PPV absorption region for $\mathrm{CyBl}$ devices.

The observation that hole transfer does not occur for $\mathrm{CyBs}$ points to differences between the HOMO energy levels for $\mathrm{CyBl}$ and CyBs. In solution, these energies are equal (Fig. 1c). However, in the solid state, the absorbance maxima are shifted to longer wavelengths by more than $50 \mathrm{~nm},{ }^{39}$ and these red-shifts must be accompanied by cooperative upward or downward shifts of the cyanine energy levels. Changes in local electronic polarization and intermolecular interactions, such as differences in the electronic structure at the interface due to dipole layer formation or charge screening effects relative to the bulk are known to affect the energy levels in the solid material. ${ }^{41,42}$ Our experiments suggest that the HOMO energy of $\mathrm{CyBl}$ in the film is shifted to lower levels, in contrast to CyBs. This then allows for charge generation via hole transfer, as apparent from the photocurrent in Fig. 6. However, an increase of $E_{\mathrm{HH}}$ implies a decrease in $E_{\Delta}$, which is reflected in a decrease of $V_{\text {oc }}$ for the MEH-PPV : CyBl system. This was indeed observed.

Estimated values for $V_{\mathrm{oc}}$ from $E_{\Delta}$ in solution are $1.2 \mathrm{~V}$ for CyA and CyC devices, and $0.9 \mathrm{~V}$ for CyBs and CyBl (Fig. 1c). The measured values at half-sun AM1.5 simulated white light $\left(47 \mathrm{~mW} \mathrm{~cm}^{-2}\right.$ ) were $1.02 \mathrm{~V}(\mathrm{CyA}), 0.98 \mathrm{~V}(\mathrm{CyC}), 0.84 \mathrm{~V}$ (CyBs) and $0.40 \mathrm{~V}(\mathrm{CyBl})$, respectively. For $\mathrm{CyA}$ and $\mathrm{CyC}$, $V_{\text {oc }}$ differs by approximately $200 \mathrm{mV}$ from $E_{\Delta}$, an offset close to that reported for other organic heterojunction devices. ${ }^{8,43}$ For CyBs, $V_{\mathrm{oc}}$ is close to the solution value $E_{\Delta}$, while for $\mathrm{CyB}$, $V_{\text {oc }}$ is substantially lower, in agreement with the necessity that efficient hole transfer needs a substantial energy gap $E_{\mathrm{HH}}$ and the observation of photocurrent generation in the region where CyBl absorbs.

\section{Conclusion}

Our results point out the merits, and some of the issues, of using cyanine dyes for photovoltaic applications. We showed that simple cw fluorescence spectroscopy can be used to estimate the charge transfer quantum yield in donor-acceptor blends and validated the results by transient absorption measurements. The concomitance of unit exciton dissociation quantum yield and low photon-to-electron conversion efficiency clearly indicates charge transport problems as the bottleneck for device performance. Doping of the cyanine has lead to substantial efficiency improvement only recently. ${ }^{44}$ The problem of nearly matched HOMO energy levels between MEH-PPV and the dyes we used here can be alleviated by synthesizing cyanines with substituents that primarily shift the energy levels but not the band gap. Our steady state PL experiments indicate that self-quenching is not dominant in blends when using low-to-medium dye concentrations, a situation that applies for actual bulk heterojunctions solar cells. As we noted above, concentrated blends of MEH-PPV and cyanines are incompatible and phase separate on large scales during spin coating. In ongoing work we therefore use semiconducting polyelectrolytes as electron donors to increase the compatibility with the ionic dyes. This allows the fabrication of bulk heterojunction devices, thereby avoiding self-quenching phenomena and making use of the advantages of the blend over the planar bilayer configuration at the same time.

\section{Experimental}

MEH-PPV with an average molecular weight of $M_{n}=$ 40000-70000 was purchased from Aldrich. Cyanine dye CyA (Fig. 1a) was purchased from Synthon Chemicals, Germany, and recrystallized from ethylacetate before use. Cyanines CyBs, CyBl and CyC (Fig. 1a) were synthesized in our laboratory. ${ }^{39,45}$ PEDOT:PSS (from Bayer), polystyrene (PS) (from Fluka), chlorobenzene (from Fluka) and 2,2,3,3-tetrafluoro-1-propanol (TFP, from Fluka) were used as received.

The redox potentials of the cyanine dyes were measured in solution by cyclic voltammetry using an Autolab potentiostat and a standard three-electrode cell. Details of the measurements are described elsewhere. ${ }^{39}$ HOMO and LUMO energy levels of MEH-PPV and PEDOT were adopted from the literature. $^{46,47}$

Thin films of MEH-PPV or PS: cyanine dye blends were fabricated on glass using different concentrations of dye (mol of dye per gram of polymer): $2.25 \times 10^{-3}$ (blend 1 ), $6.40 \times 10^{-4}(2), 1.65 \times 10^{-4}(3), 4.65 \times 10^{-5}(4), 1.50 \times 10^{-5}(5)$, $4.50 \times 10^{-6}(6)$ and $1.50 \times 10^{-6}(7)$. This corresponds to $60 \%, 30 \%, 10 \%, 3 \%, 1 \%, 0.3 \%$ and $0.1 \%$ in weight $(\mathrm{wt} \%$ ) for the CyA:polymer blends. Absorbance measurements were made with a Varian Cary $50 \mathrm{UV}$-Vis spectrophotometer. Fluorescence measurements were performed on a Fluorolog-3 spectrofluorometer (Jobin Yvon Horiba) in front-face detection mode. All spectra were corrected by the percentage of light absorbed at the excitation wavelength. Since no integrating sphere was used, the presented 
photoluminescence values can only be interpreted relative to each other.

Photovoltaic devices were fabricated on ITO-coated glass from Merck with a sheet resistance of $30 \Omega / \square$ covered by a $80 \mathrm{~nm}$ thick PEDOT layer. After heating at $120{ }^{\circ} \mathrm{C}$ under vacuum for $90 \mathrm{~min}$, the substrates were transferred to a nitrogen-filled glove box. Then, a MEH-PPV film with a thickness of approximately $30 \mathrm{~nm}$ was spin coated from a $5 \mathrm{mg} \mathrm{mL}^{-1}$ chlorobenzene solution onto PEDOT. On top of this thin film, the dye layers were spin coated with a thickness of $45-50 \mathrm{~nm}$ from a solution of $5 \mathrm{mg}$ of cyanine dissolved in $1 \mathrm{~mL}$ of a solvent mixture of TFP: chlorobenzene $=6: 1$ by volume. A small amount of chlorobenzene had to be added so that the solution would wet the polymer layer and form a homogeneous dye film. In order to be sure that the underlying MEH-PPV layer was not dissolved, we checked the film by both optical microscopy and by measuring the UV-vis spectra before and after spin coating the solvent mixture only (without dye) on top of the polymer film. No change could be observed, which led us to conclude that the polymer film was not affected by the solvent mixture of TFP: chlorobenzene.

Finally, a $40 \mathrm{~nm}$ thick aluminum cathode layer was thermally deposited under high vacuum $\left(<3 \times 10^{-6} \mathrm{mbar}\right)$ to form active device areas of 0.07 or $0.03 \mathrm{~cm}^{2}$. Film thicknesses were measured with an Ambios Technology XP-1 surface profilometer. Solar cells were stored and subsequently analyzed in a $\mathrm{N}_{2}$ atmosphere. The spectrally-resolved photocurrents under short-circuit conditions were measured using a monochromator with a xenon lamp light source having an irradiation intensity of approximately $1 \mathrm{~mW} \mathrm{~cm}^{-2}$ at $500 \mathrm{~nm}$. Open-circuit voltages $\left(V_{\mathrm{oc}}\right)$ and short-circuit currents $\left(J_{\mathrm{sc}}\right)$ were measured under simulated AM1.5 white light (47 $\mathrm{mW} \mathrm{cm}^{-2}$ ) using a Keithley 2400 source-measure unit. Details on the setup for photocurrent measurements were described elsewhere. ${ }^{48}$

For nanosecond transient absorption measurements, pulsed laser excitation was applied using a broadband optical parametric oscillator (OPO), pumped by a frequency-tripled Q-switched Nd:YAG laser $(30 \mathrm{~Hz}$ repetition rate, pulse width at half-height $5 \mathrm{~ns}$ ). The output of the OPO was tuned at $\lambda=650 \mathrm{~nm}$ and attenuated by grey filters. The beam was expanded by a plano-concave lens to irradiate a large crosssection $\left(\sim 1 \mathrm{~cm}^{2}\right)$ of the sample, whose surface was kept at a $30^{\circ}$ angle to the excitation beam. The analyzer light, produced by a cw Xe arc lamp, was passed through a monochromator, various optical elements, the sample and a second monochromator, prior to being detected by a fast photomultiplier tube. Satisfactory signal-to-noise ratios were typically obtained by averaging over several hundred laser shots.

\section{Acknowledgements}

We thank Thomas Geiger (Empa) for the synthesis of the cyanine dyes.

\section{References}

1 C. J. Brabec, Sol. Energy Mater. Sol. Cells, 2004, 83, 273.

2 S. E. Gledhill, B. Scott and B. A. Gregg, J. Mater. Res., 2005, 20, 3167.
3 H. Spanggaard and F. C. Krebs, Sol. Energy Mater. Sol. Cells, 2004, 83, 125.

4 S. Günes, H. Neugebauer and N. S. Sariciftci, Chem. Rev., 2007, 107,1324

5 G. Li, V. Shrotriya, J. Huang, Y. Yao, T. Moriarty, K. Emery and Y. Yang, Nat. Mater., 2005, 4, 864.

6 J. Xue, B. P. Rand, S. Uchida and S. R. Forrest, Adv. Mater., 2005, 17,66 .

7 A. Gadisa, M. Svensson, M. R. Andersson and O. Inganäs, Appl. Phys. Lett., 2004, 84, 1609.

8 M. C. Scharber, D. Mühlbacher, M. Koppe, P. Denk, C. Waldauf, A. J. Heeger and C. J. Brabec, Adv. Mater., 2006, 18, 789.

9 R. B. Ross, C. M. Cardona, D. M. Guldi, S. G. Sankaranarayanan, M. O. Reese, N. Kopidakis, J. Peet, B. Walker, G. C. Bazan, E. van Keuren, B. C. Holloway and M. Drees, Nat. Mater., 2009, 8, 208.

10 B. P. Rand, D. P. Burk and S. R. Forrest, Phys. Rev. B: Condens. Matter Mater. Phys., 2007, 75, 115327.

11 F. Zhang, E. Perzon, X. Wang, W. Mammo, M. R. Andersson and O. Inganäs, Adv. Funct. Mater., 2005, 15, 745.

12 X. Wang, E. Perzon, W. Mammo, F. Oswald, S. Admassie, N.-K. Persson, F. Langa, M. R. Andersson and O. Inganäs, Thin Solid Films, 2006, 511-512, 576.

13 C. Soci, I. W. Hwang, D. Moses, Z. Zhu, D. Waller, R. Gaudiana, C. J. Brabec and A. J. Heeger, Adv. Funct. Mater., 2007, 17, 632.

14 E. Bundgaard and F. C. Krebs, Sol. Energy Mater. Sol. Cells, 2007, 91, 954.

15 F. Meng, J. Hua, K. Chen, H. Tian, L. Zuppiroli and F. Nüesch, Proc. SPIE-Int. Soc. Opt. Eng., 2005, 5938, 593814.

16 N. A. Davidenko, N. A. Derevyanko, A. A. Ishchenko, N. G. Kuvshinsky, A. V. Kulinich, O. Y. Neiland and M. V. Plotniece, Russ. Chem. Bull., 2004, 53(8), 1674.

17 S. Dhami, A. J. De Mello, G. Rumbles, S. M. Bishop, D. Phillips and A. Beeby, Photochem. Photobiol., 1995, 61, 341.

18 T. D. Slavnova, A. K. Chibisov and H. Görner, J. Phys. Chem. A, 2002, 106, 10985.

19 M. Krieg and R. W. Redmond, Photochem. Photobiol., 1993, 57, 472.

20 T. H. James, The Theory of the Photographic Process, Macmillan, New York, 4th edn, 1977.

21 K. Sayama, K. Hara, Y. Ohga, A. Shinpou, S. Suga and H. Arakawa, New J. Chem., 2001, 25, 200.

22 A. V. Buettner, J. Chem. Phys., 1967, 46, 1398.

23 A. A. Ishchenko, Theor. Exp. Chem., 1998, 34(4), 191.

24 H. Benmansour, F. A. Castro, M. Nagel, J. Heier, R. Hany and F. Nüesch, Chimia, 2007, 61, 787.

25 M. Yan, L. J. Rothberg, E. W. Kwock and T. M. Miller, Phys. Rev. Lett., 1995, 75, 1992.

26 J. Liu, Y. Shi and Y. Yang, Appl. Phys. Lett., 2001, 79, 578.

27 A. Mishra, R. K. Behera, P. K. Behera, B. K. Mishra and G. B. Behera, Chem. Rev., 2000, 100, 1973.

28 J. R. Lenhard and A. D. Cameron, J. Phys. Chem., 1993, 97, 4916.

29 Y. Huang, T. Cheng, F. Li, C. Luo, C.-H. Huang, Z. Cai, X. Zeng and J. Zhou, J. Phys. Chem. B, 2002, 106, 10031.

30 A. Mishra and N. S. Haram, Dyes Pigm., 2004, 63, 191.

31 R. F. Khairutdinov and N. Serpone, J. Phys. Chem. B, 1997, 101, 2602.

32 C. Peyratout, E. Donath and L. Daehne, J. Photochem. Photobiol., $A, 2001,142,51$.

33 A. Gilbert and J. Baggot, Essentials of Molecular Photochemistry, Blackwell, 1st edn, 1991.

34 D. E. Markov, E. Amsterdam, P. W. M. Blom, A. B. Sieval and J. C. Hummelen, J. Phys. Chem. A, 2005, 109, 5266.

35 B. Kraabel, J. C. Hummelen, D. Vacar, D. Moses, N. S. Sariciftci, A. J. Heeger and F. Wudl, J. Chem. Phys., 1996, 104, 4267.

36 L. P. Candeias, F. C. Grozema, G. Padmanaban, S. Ramakrishnan, L. D. A. Siebbeles and J. M. Warman, J. Phys. Chem. B, 2003, 107, 1554.

37 H. D. Burrows, M. G. Miguel, A. P. Monkman, I. Hamblett and S. Navaratnam, J. Mol. Struct., 2001, 563-564, 41.

38 Y. H. Kim, D. Kim, S. C. Jeoung, J. Y. Han, M. S. Jang and H. K. Shim, Chem. Mater., 2001, 13, 2666.

39 F. A. Castro, A. Faes, T. Geiger, C. F. O. Graeff, M. Nagel, F. Nüesch and R. Hany, Synth. Met., 2006, 156, 973. 
40 A. M. Ramos, E. H. A. Beckers, T. Offermans, S. C. J. Meskers and R. A. J. Janssen, J. Phys. Chem. A, 2004, 108, 8201.

41 H. Ishii, K. Sugiyama, E. Ito and K. Seki, Adv. Mater., 1999, 11, 605.

42 S. C. Veenstra and H. T. Jonkman, J. Polymer Sci. B, 2003, 41, 2549.

43 D. Veldman, J. J. A. M. Bastiaansen, B. M. W. Langeveld-Voss, J. Sweelssen, M. M. Koetse, S. C. J. Meskers and R. A. J. Janssen, Thin Solid Films, 2006, 511-512, 581.
44 B. Fan, R. Hany, J.-E. Moser and F. Nüesch, Org. Electron., 2008, 9, 85 .

45 F. Nüesch, A. Faes, L. Zuppiroli, F. Meng, K. Chen and H. Tian, J. Mater. Sci., 2005, 40, 1353.

46 M. M. Alam and S. A. Jenekhe, Chem. Mater., 2004, 16, 4647.

47 F. Nüesch, G. Tornare, L. Zuppiroli, F. Meng, K. Chen and H. Tian, Sol. Energy Mater. Sol. Cells, 2005, 87, 817.

48 F. A. Castro, H. Benmansour, C. F. O. Graeff, F. Nüesch, E. Tutis and R. Hany, Chem. Mater., 2006, 18, 5504. 